\title{
Status report and performance of the T2K experiment
}

\section{Eike FRANK* for the T2K collaboration}

Laboratory for High Energy Physics (LHEP), University of Bern, Switzerland

E-mail: eike.frankelhep.unibe.ch

\begin{abstract}
$\mathrm{T} 2 \mathrm{~K}$ is the first long baseline off-axis neutrino oscillation experiment and aims to refine the measurements of the oscillation parameters $\Delta m_{23}^{2}$ and $\theta_{23}$, discovered by Super-Kamiokande with atmospheric neutrinos, respectively at a level of $10 \%$ and $<1 \%$ uncertainties in disappearance neutrino oscillation mode. Moreover, these parameters are fundamental ingredients for the key research item of T2K that is the discovery of $\theta_{13}$ by measuring the appearance of electron neutrinos, which is furthermore needed to understand CP-violation in the leptonic sector. This will be done by comparing the flux of a high intensity neutrino beam at a near detector station, ND280 composed of on-axis and $2.5^{\circ}$ off-axis detectors at roughly $280 \mathrm{~m}$ from the neutrino source) with the flux $2.5^{\circ}$ off-axis at the far detector, Super-Kamiokande ( $295 \mathrm{~km}$ from the source).
\end{abstract}

Identification of Dark Matter 2010

July 26 - 302010

University of Montpellier 2, Montpellier, France

\footnotetext{
*Speaker.
} 


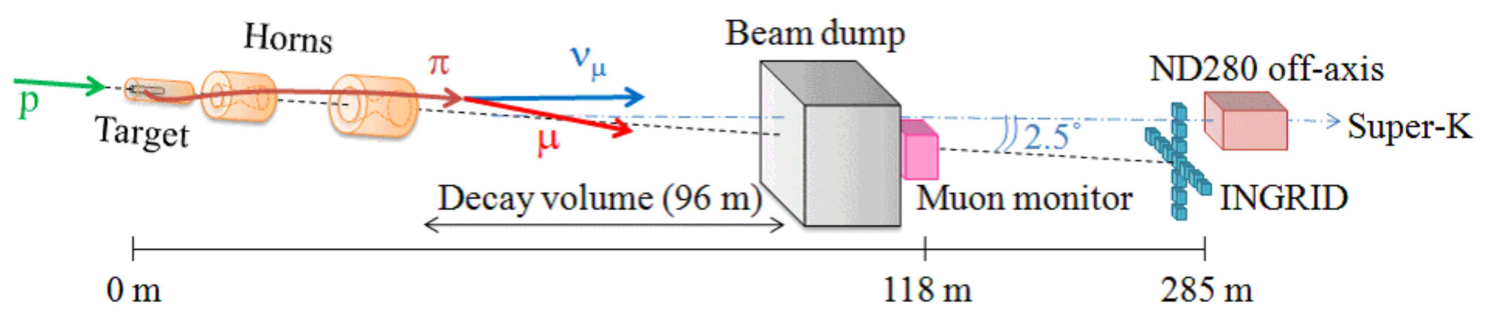

Figure 1: The $30 \mathrm{GeV}$ proton beam, produced at J-PARC, hits the carbon target and thereby produces secondary Mesons, mainly pions, which are focused by the three horns. The pions decay further and thus produce a high intensity neutrino beam which is monitored by several near detectors before traveling further towards Super-Kamiokande at an off-axis angle of $2.5^{\circ}$.

\section{Introduction}

The T2K experiment [1] is the first long baseline neutrino oscillation experiment running with an intense off-axis $v_{\mu}$ beam peaking at an energy of $600 \mathrm{MeV} / \mathrm{c}$. This beam is produced at J-PARC, a newly built high intensity proton accelerator complex in Tokai, Japan. After traveling $295 \mathrm{~km}$, the neutrinos are observed in a water Cherenkov detector, Super-Kamiokande at Kamioka, Japan. The ND280 near detector complex, installed at a distance of $280 \mathrm{~m}$ from the neutrino source, measures the beam properties before oscillations.

In the three generations framework, neutrino oscillation probabilities can be written as:

$$
\begin{aligned}
& P_{v_{\mu} \rightarrow v_{e}} \approx \sin ^{2} 2 \theta_{13} \cdot \sin ^{2} \theta_{23} \cdot \sin ^{2} \Phi_{23}, \\
& P_{v_{\mu} \rightarrow v_{\mu}} \approx \cos ^{4} \theta_{13} \cdot \sin ^{2} 2 \theta_{23} \cdot \sin ^{2} \Phi_{23},
\end{aligned}
$$

where $\Phi_{23} \approx 1.27 \Delta m_{23}^{2} L / E_{v}$ and $\theta_{i j}$ are the mixing angles of the PMNS (Pontecorvo-MakiNakagawa-Sakata [2]) matrix.

The main physics motivations of the $\mathrm{T} 2 \mathrm{~K}$ experiment are:

1. Discovery of $v_{\mu} \rightarrow v_{e}$ oscillations measuring the only missing angle $\theta_{13}$ in the PMNS matrix through Eq. 1.1. The $v_{e}$ interactions in Super-Kamiokande will be identified by the distinctive signature of the Cherenkov light emitted by the electrons. The expected T2K sensitivity is $\sin ^{2} 2 \theta_{13}=0.006$ at $90 \%$ C.L. for normal hierarchy and assuming $10 \%$ systematic errors. This represents an improvement of more than one order of magnitude over the current limit provided by the experiments Chooz and MINOS [3], [4]. If $\theta_{13}$ is non-zero, this gives a possible window to $\mathrm{CP}$-violation in the leptonic sector in a second phase of the experiment with an even larger far detector.

2. Precision measurement of oscillation parameters in $v_{\mu}$ disappearance mode (Eq. 1.2). The goal is an observation of the oscillation minimum, with $1 \%$ measurement of $\sin ^{2} 2 \theta_{23}$ and $<10^{-4} \mathrm{eV}^{2}$ error on $\Delta m_{23}^{2}$. 
To achieve these goals, T2K will accumulate $8 \times 10^{21}$ POT (Protons On Target) which corresponds to 5 years of running with the design beam power of $0.75 \mathrm{MW}$. A key element of the T2K setup is the off-axis neutrino beam, which means that the beam axis misses Super-Kamiokande by $2.5^{\circ}$. The reason for this feature is related to the kinematics of the pion decay: pions in a wide momentum range contribute to a narrower energy spread for neutrinos. This results in a considerable improvement in the quality of the beam for the measurement of oscillation parameters as it is centered to the expected oscillation maximum.

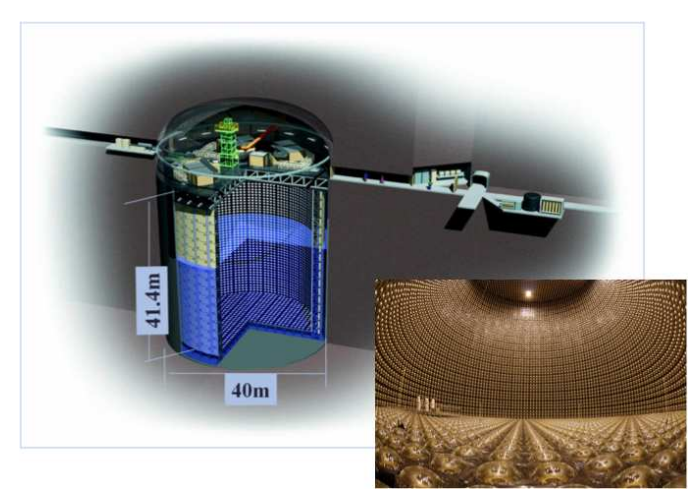

Figure 2: The Super-Kamiokande detector as an artistic drawing and a photo from the inside showing a big part of the 11,129 PMTs.

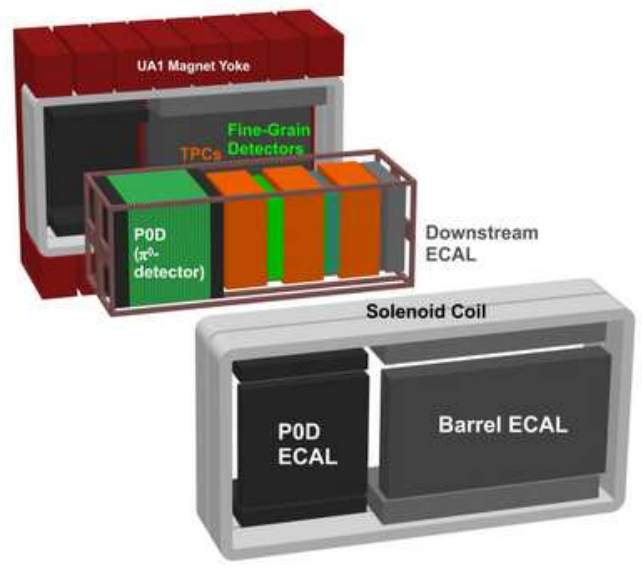

Figure 3: The sub-detectors of ND280. The neutrino beam is advancing from the left and passing through several detectors with various tasks in order to estimate the neutrino beam energy spectra and the cross-sections for neutrino interaction in the enrgy region around $1 \mathrm{GeV}$.

\section{Experimental Setup}

The beamline: To produce the neutrino beam, a train of six bunches of $30 \mathrm{GeV} / \mathrm{c}$ protons is extracted from the Main Ring every $3.5 \mathrm{~s}$. The protons are transported to the graphite target: the intensity, position, profile and beam loss of the proton beam are monitored by five current transformers, 21 electrostatic monitors, 19 segmented secondary emission monitors, an optical transition radiation monitor and 50 beam loss monitors. The collision between the protons and the target produce hadrons (Figure1) that are focused by three magnetic horns with $250 \mathrm{kA}$ pulsed currents. The hadrons enter the decay volume where they decay mainly into muons and muon neutrinos. All the remnants of the undecayed pions and other hadrons are stopped by a beam dump. This produces a pure beam of $v_{\mu}$ with an expected $v_{e}$ contamination of $1.5 \%$ ( $0.4 \%$ at the oscillation maximum). Downstream the beam dump, the flux and direction of the muons coming from the pion decays are measured spill by spill by the Muon Monitor (MUMON).

Super-Kamiokande: Super-Kamiokande is a $22.5 \mathrm{kt}$ fiducial mass ring-imaging water Cherenkov detector located at a depth of 2700 meters water equivalent in the Kamioka mine (Figure 2). The 


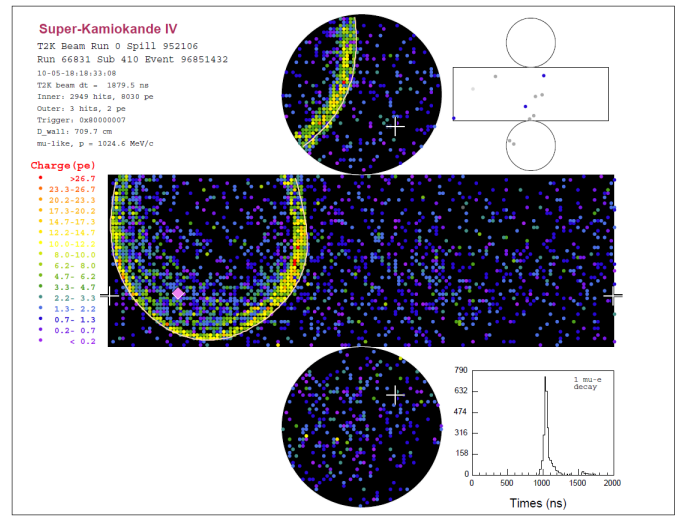

Figure 4: The event display shows an 1-single ring $\mu$-like example event at Super-Kamiokande.

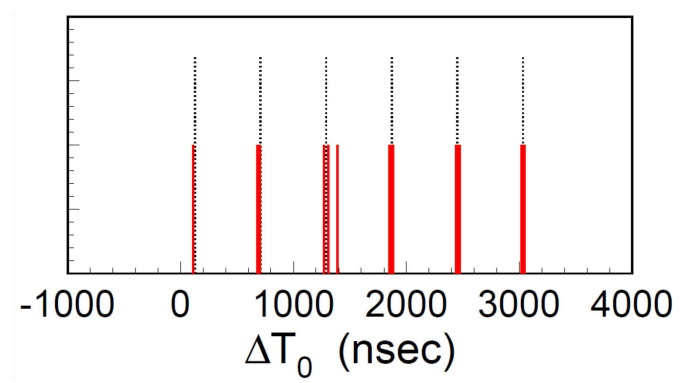

Figure 5: The timing of the candidate events matches very well with the bunch structure of the beam, thus rejecting the non-beam background.

detector is optically separated into two concentric cylindrical regions: the inner detector instrumented with 11,129 20-inch photomultiplier tubes (PMT) and the outer detector with 1885 8-inch PMTs. A new readout electronics system has been installed for T2K and the synchronization with the J-PARC beam is achieved with a GPS system. Super-Kamiokande has good capability to identify muons and electrons by analyzing the sharpness of a Cherenkov ring. A muon makes a sharp edge ring and an electron makes a fuzzy one due to electromagnetic showers. The electron/muon misidentification probability is about $1 \%$ for the T2K neutrino energy. $v_{e}$ appearance signals can be seen by selecting one-ring electron-like events in charged current quasi elastic (CCQE) samples. Backgrounds arise from the intrinsic $v_{e}$ contamination of the beam and misreconstructed $\pi^{0}$ events in which the e-like rings are not separated because the opening angle is to small or because the $\pi^{0}$ decay is asymmetrical and the low energy photon is not detected: both backgrounds can be measured at ND280. The expected signal over background ratio is 5.5(0.5) for $\sin ^{2} 2 \theta_{13}=0.1(0.01)$.

The Near Detector: The Near Detector complex consists of an on-axis detector (INGRID) and an off-axis detector. INGRID is composed by 14 modules of alternated iron and plastic scintillator planes and counts the number of neutrino charged interactions in each module to reconstruct the profile and direction of the beam. The off-axis ND280 (Figure 3) consists of several detectors installed in the former UA1 magnet operated at $0.2 \mathrm{~T}$ : a $\pi^{0}$ detector (P0D) to measure interactions with $\pi^{0}$ production, an electromagnetic calorimeter (ECal) surrounding the inner region of ND280 to measure the electromagnetic activity and a Side Muon Range Detector (SMRD) embedded in the magnet yokes to measure muons escaping from the side. All these detectors use Multi-Pixel Photon Counters [5]. Finally a tracker system, composed by 2 Fine Grained Detectors (FGD) and 3 Time Projection Chambers (TPC) instrumented with MicroMegases [6] will measure $v_{\mu}$ and $v_{e}$ fluxes, reconstructing the lepton momentum from the curvature and separating electrons from muons using $\mathrm{dE} / \mathrm{dx}$ according to the Bethe-Bloch fromula. 


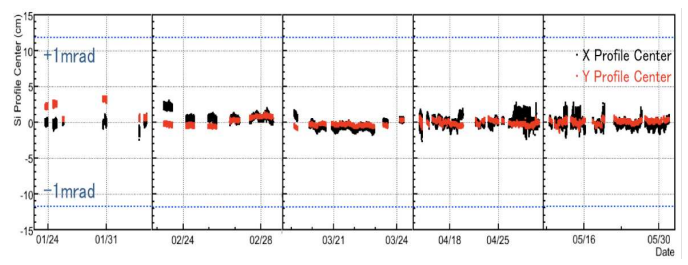

Figure 6: The MUMON detector monitors the the beam on a spill by spill basis, showing that the angular spread is well within the required limit of $1 \mathrm{mrad}$.

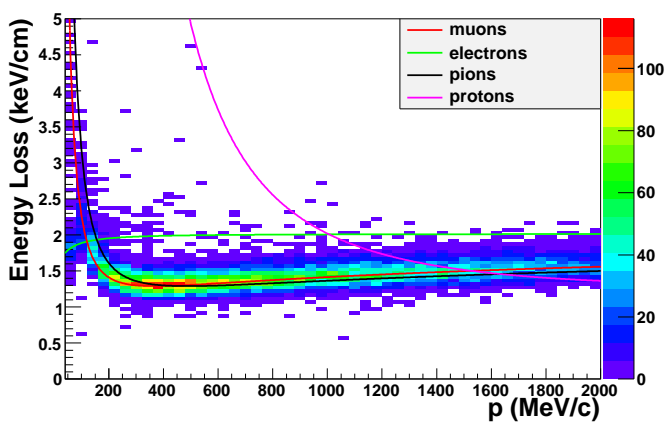

Figure 8: Energy loss as a function of the reconstructed momentum in TPCs for negative tracks.

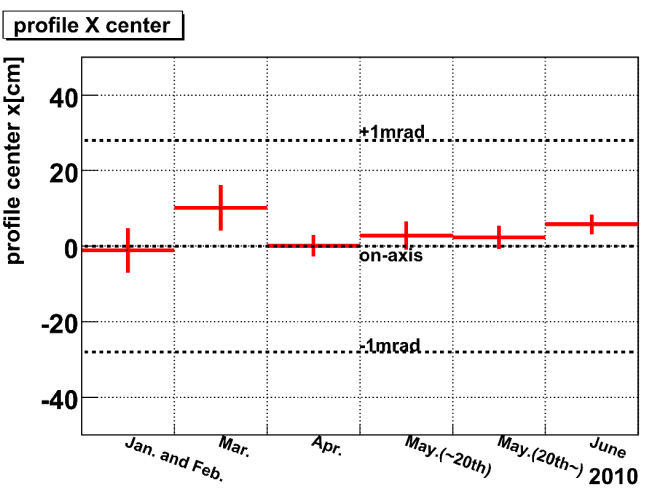

Figure 7: INGRID measures the on-axis neutrino beam which stable within the required limit of $1 \mathrm{mrad}$.

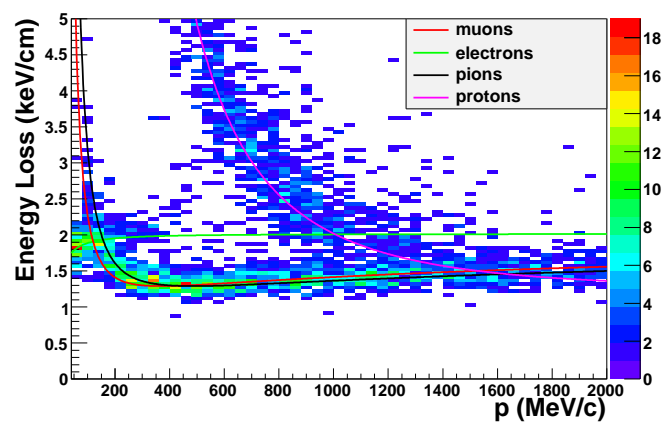

Figure 9: Energy loss as a function of the reconstructed momentum in TPCs for positive tracks.

\section{First results from the 2010 physics run}

The T2K first physics run started in January 2010 and was completed in June 2010. A total of $3.35 \times 10^{19}$ POT was delivered, running at a continuous beam power of $50 \mathrm{~kW}$. During this run all detectors were operating, except the Barrel ECAL which was installed in Fall 2010. The livetime was $>99 \%$ at Super-Kamiokande and 96\% at ND280. 23 fully contained events were observed in the fiducial volume of Super-Kamiokande. One single ring $\mu$-like event observed in SuperKamiokande is shown in Figure 4. Figure 5 shows the time distributions of the events observed in Super-Kamiokande compared to the expected times for the six beam bunches: 26 ns resolution in beam timing synchronization has been reached thanks to the GPS system. The beam direction and profile were monitored by MUMON (Figure 6) and by INGRID (Figure7) during the first physics run: the beam direction is well within the $1 \mathrm{mrad}$ required tolerance. In off-axis ND280 thousands of interactions have been observed: the reconstructed momentum of the tracks crossing the TPCs and their energy loss are shown in Figure 8 and 9 for negative and positive tracks respectively: these plots show the capability of ND280 of measuring the $v_{\mu}$ and $v_{e}$ energy spectra by distinguishing muons from electrons and reconstructing their momenta. 


\section{Summary}

T2K completed its first physics run in June 2010. During this run a total of $3.35 \times 10^{19}$ POT have been delivered and all the detectors are working as expected. The first measurements of the oscillation parameters, using this data set, are expected shortly.

\section{References}

[1] Y. Itow et. al., hep-ex/0106019

[2] Z. Maki, M. Nagakawa and S. Sakata, Prog. Theor. Phys. 28, 870 (1962)

[3] M. Apollonio et al., Eur. Phys. J. C 27, 331 (2003)

[4] P. Adamson et al. [The MINOS Collaboration], arXiv:1006.0996v1 [hep-ex]

[5] M. Yokoyama et al., Application of Hamamatsu MPPC to T2K Neutrino Detectors, Nucl. Instrum. Meth. A 610 (2009) 128

[6] I. Giomataris et al., Micromegas in a Bulk, Nucl. Instr. Meth. A 560 405, 2006 